\title{
Tuning the strength of the resonance-assisted hydrogen bond in acenes and phenacenes with two o- hydroxyaldehyde groups. The importance of topology
}

Gerard Pareras ${ }^{a, \ddagger}$, Dariusz W. Szczepanik ${ }^{b, \ddagger}$, Miquel Duran ${ }^{b}$, Miquel Solàb,* and Sílvia Simon ${ }^{\mathrm{b}, *}$

a Department of Chemistry, University College Cork, College Rd, Cork, Ireland

b Institut de Química Computacional i Catàlisi and Departament de Química, Universitat de Girona, C/ Maria Aurèlia Capmany 69, 17003 Girona, Catalonia, Spain

¥ These two authors contributed equally to this work.

Corresponding authors: silvia.simon@udg.edu, miquel.sola@udg.edu

\section{TOC Graphic}

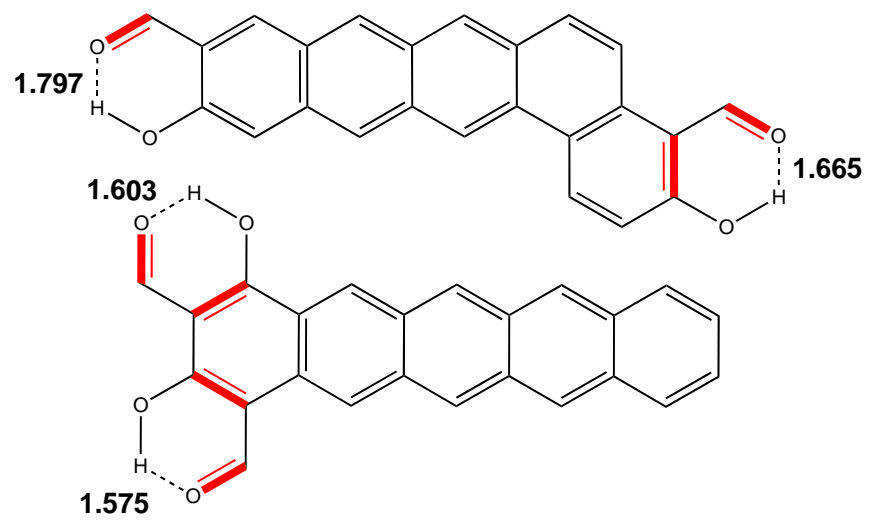




\section{Abstract}

The fact that intramolecular resonance-assisted hydrogen bonds (RAHBs) are stronger than conventional ones is attributed to the partial delocalization of the $\pi$ electrons within the hydrogen bond $(\mathrm{HB})$ motif, the so-called quasi-ring. If an aromatic ring is involved in the formation of the RAHB, previous studies have shown that there is an interplay between aromaticity and HB strength. Moreover, in 1,3-dihydroxyaryl-2-aldehydes, some of us found that the position of the quasiring formed by the substituents interacting through RAHB influences the strength of the $\mathrm{H}$-bonding, the HBs being stronger when a kinked-like structure is generated by formation of the quasi-ring. In this work, we explore this concept further by considering a set of acenes and phenacenes of different sizes with two 0 hydroxyaldehyde substituents. Calculations with the CAM-B3LYP/6$311+G(d, p)+G D 3 B$ method show that for long acenes or phenacenes, once the substituent effect loses importance because quasi-rings are pull apart far from each other, the different topologies rule the HB distances. This fact can be explained in most cases using an extended Clar's aromatic $\pi$-sextet model. In some kinked systems, however, the justification from the Clar model has to be complemented by taking into account the repulsion between hydrogen atoms. Triphenylene-like compounds with different number of benzene rings have been studied finding out a very good relationship between aromaticity of the ipso- and quasi-rings with the RAHB distances. This result confirms the importance of the communication of the $\pi$-systems of the ipso- and quasi-rings. 


\section{Introduction}

Hydrogen bonds (HBs) are one of the strongest non-covalent interactions. They play an important role in many chemical and biological processes; for instance, HBs determine the crystal structure as well as the stabilization of the second-order structure of proteins. ${ }^{1-4}$ Because of their directionality and the fact of being strong enough to assemble molecular systems in aggregates, HBs are one of the most important interactions in supramolecular chemistry and molecular engineering. ${ }^{5}$ Depending on its bond strength, which can range from $0.2 \mathrm{kcal} / \mathrm{mol}$ to $40 \mathrm{kcal} / \mathrm{mol}$, HBs can be classified as weak, moderate or strong. ${ }^{2}$

HBs assisted by additional effects, e.g., resonance-assisted or charge-assisted, ${ }^{6-}$ 9 are among the strongest HBs known. Intramolecular resonance-assisted hydrogen bonds (RAHB) introduced by Gilli et al. ${ }^{6-9}$ are stronger than conventional HBs because of the extra stabilization connected with the partial delocalization of the $\pi$-electrons within the so-called quasi-ring containing conjugated formally single and double bonds. A schematic representation of electronic effects proceeding within such cyclic RAHB is shown in Scheme 1 for malonaldehyde. The characteristic elongation of formally double bond and shortening of formally single bonds in the quasi-ring together with a strengthening of the $\mathrm{HB}$ are fingerprints of RAHBs.

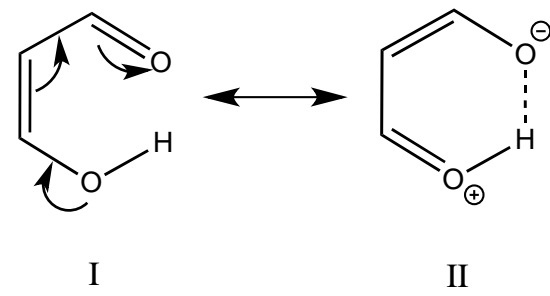

Scheme 1. The two main resonance structures involved in the resonance-assisted hydrogen bond in malonaldehyde.

Many computational studies ${ }^{10-27}$ and experimental works ${ }^{28-31}$ can be found related to intramolecular and intermolecular RAHBs. The origin of the additional strength of RAHB as compared to conventional HBs is still subject of an ongoing debate. 
Gilli et al..$^{6-9}$ attributed it to the delocalization of the $\pi$-electrons. Mo et al, ${ }^{16,18}$ provided further evidences that the enhanced HB comes from the charge flow from the HB donor to the acceptor through the $\pi$-conjugation. Recently, Grosch et al. ${ }^{11}$ arrived to the conclusion that $\pi$ polarization and $\sigma$ charge transfer are the responsible for enhancing RAHB in line with the Gilli et al. proposal, although they emphasized that there is no resonance assistance in the sense of an interplay between $\sigma$ charge transfer and $\pi$ polarization. Moreover, other researchers, like Sanz et al., ${ }^{25,26}$ argued that the characteristics of the $\sigma$-skeleton, and not the resonance assistance phenomenon, is the main responsible for the extra stability of RAHB. Also, Guevara-Vela et al.13,14,24 analyzed the nature of the RAHB to conclude that there is an increase in electron localization of $\pi$-electrons (not delocalization as expected from Gilli's interpretation) in the quasi-ring of RAHB. This localization leads to greater electrostatic, polarization, and charge transfer effects that result in stronger $\mathrm{H}$-bonds. Thus, we can find different interpretations of the basis of the "assistance" in RAHBs.
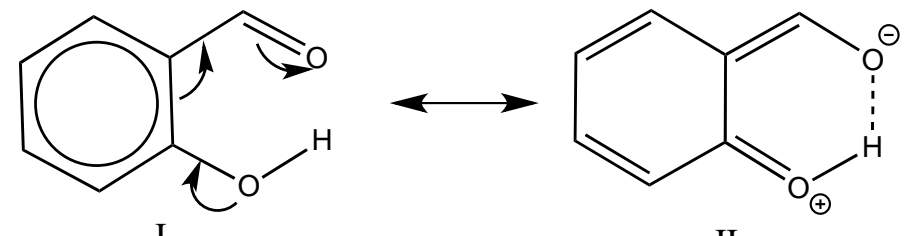

II

Scheme 2. The two main resonance structures involved in the resonance-assisted hydrogen bond in o-hydroxybenzaldehyde.

The first relation between HB formation and aromaticity was reported in a landmark paper by Dewar already in $1945 .{ }^{32}$ In his study, Dewar ${ }^{32}$ showed that presence of an intramolecular hydrogen bond induces aromatic character at the sevenmembered ring (7-MR) of the stipatic acid, thus providing an explanation to the physicochemical properties of this molecule. More recently, studies by our group $^{33,34}$ described the interplay between RAHB strength and aromaticity of the ipso-ring in a series of o-hydroxyarylaldehydes (see Scheme 2). These compounds can separate the $\pi$-resonance effect in three different individual 
effects, namely, (a) the delocalization within the quasi-ring, identified by Gilli et al. ${ }^{8}$ and already present in malonaldehyde, (b) the effect of the $\pi$-electron delocalized within the benzene ring (structure I in Scheme 2), and (c) the substituent effect of hydroxyl group (electron-donating group) and carbonyl (electron-withdrawing) that favors the canonical structure with charge separation (structure II in Scheme 2). Compared to malonaldehyde, with $\mathrm{C}=\mathrm{C}$ localized double bond, effects (a) and (c) cooperate with each other, so reinforcing the HB, while (b) goes against HB formation. From the data found in these previous studies, it is clear that RAHB enhancement or weakening can be controlled by changing the substituents ${ }^{35}$ and the aromaticity of ipso-ring. ${ }^{33,34}$ Indeed, in 1,3-dihydroxyaryl-2-aldehydes, ${ }^{33}$ we found that the position of the quasi-ring formed by the substituents interacting through RAHB influences the strength of the $\mathrm{H}$-bonding, the HBs being stronger when a kinked-like structure is generated by formation of the quasi-ring. Similar results were obtained by Houjou et al. ${ }^{28,29}$ from the analysis of the proton transfer processes in the two systems represented in Scheme 3. These authors showed that HBs are stronger for I than II irrespective of solvent polarity or crystal packing. According to the authors, the reason is the low distortion of the aromatic rings in $\mathbf{I}$ as compared to II. For the latter, the loss of aromaticity is higher reducing the stability of the HBs formed.
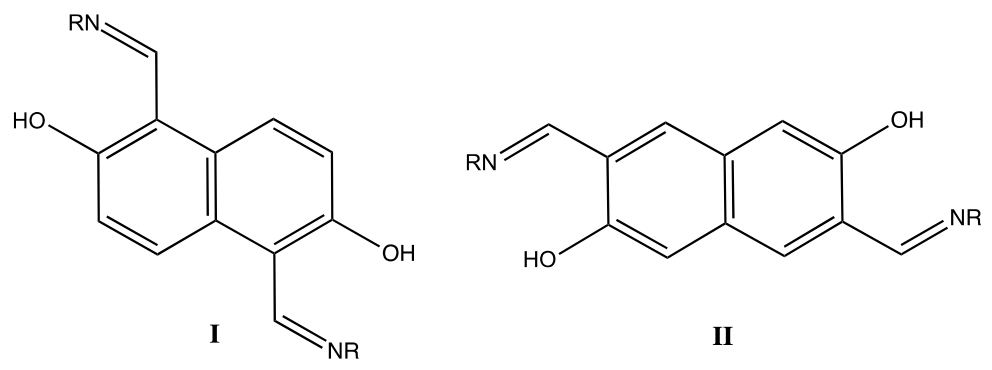

Scheme 3. Structure of the $\alpha, \alpha$-diimine (I) and $\beta, \beta$-diimine (II) species.

It is very well-known that aromaticity of condensed benzene rings, the so-called benzenoid hydrocarbons, depends on their number of rings as well as their relative position, that is, their isomeric structure. [ $n]$ Acenes and $[n]$ phenacenes ( $n$ being the number of rings) are two series of isomeric aligned benzenoid compounds 
(Scheme 4). The aromaticity of these compounds has been widely analyzed using different indices. ${ }^{36}$ This aromaticity can be explained using Clar's $\pi$-sextet model, ${ }^{37}$ which states the Kekule resonance structure with the largest number of disjoint aromatic $\pi$-sextets is the most important. Following this rule, the Clar's structures for [3]acene (anthracene) and [3]phenacene (phenanthrene) are the ones represented in Scheme 4. [n]Acenes with a migrating $\pi$-sextet have similar aromaticity in all rings, ${ }^{38}$ whereas in [n]phenacenes the outer rings are more aromatic than the central one. Moreover, [n]phenacenes have a larger number of aromatic $\pi$-sextets than the corresponding [ $n]$ acenes and, therefore, are more stable and more aromatic as a result of more stabilizing $\pi$-interactions. ${ }^{39}$

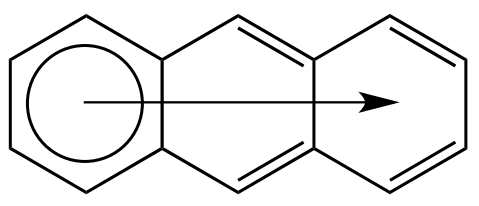

(a)

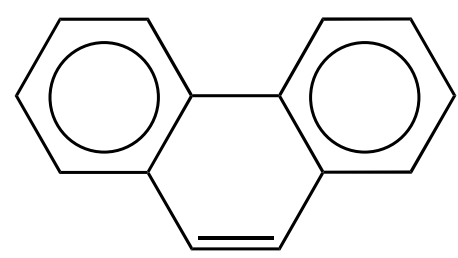

(b)

Scheme 4. Clar structures for (a) anthracene and (b) phenanthrene.

Following this line of reasoning, one could consider system II in Scheme 3 as a part of a pseudo-tetracene species formed by a naphthalene attached to two quasirings in a linear arrangement, whereas compound I as a part of pseudo-chrysene with two quasi-rings in a kinked topology. Given that [n]phenacenes are more stable than [n]acenes, one can make the hypothesis than I is more stable than II due to the better $\pi$ interactions found in kinked structures as compared to linear ones. The main goal of this work is to prove this hypothesis by analyzing a set of acenes and phenacenes of different sizes with two o-hydroxyaldehyde substituents at the outermost rings. We anticipate here that the arrangement of the aromatic rings has a great influence in the RAHB strength and that kinked distribution of rings results in stronger RAHBs. 


\section{Results and Discussion}

In this section, we will discuss first the changes in RAHB distances when increasing the chain of polycyclic aromatic hydrocarbons (PAHs) in two different topologies, linear and kinked structures. Then we will analyze the effect of including sequentially a new kink starting from a linear topology to finally yield the fully kinked structure. Finally, the design of triphenylene-like compounds will be used to discuss the direct relation between aromaticity and HB strength.

By adding an extra quasi-ring to o-hydroxybenzaldehyde (Scheme 2) formed by two adjacent $\mathrm{OH}$ and $\mathrm{HCO}$ substituents, one can generate the five different

isomers of dihydroxydibenzaldehyde shown in Figure 1. Two of them present a linear arrangement and resemble anthracene, whereas three of the isomers have a kinked structure and bear a resemblance to phenanthrene.

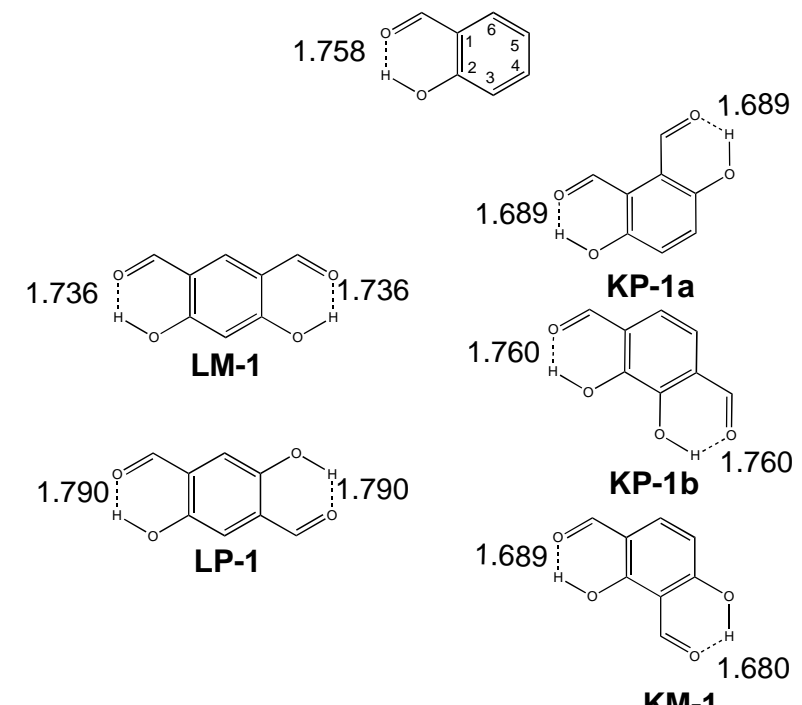

KM-1

Figure 1. Dihydroxydibenzaldehyde isomers with two quasi-rings. RAHB distances in $\AA$.

When the new substituents to build the quasi-ring $(\mathrm{OH}$ and $\mathrm{HCO})$ are added in positions 4 and 5 of $o$-hydroxybenzaldehyde (see Figure 1 for numbering), one can have two linear conformers, LM-1 and LP-1 $(L$ for linear and $M$ and $P$ respect to the relative position of both $\mathrm{OH}$ and $\mathrm{HCO}$ in the ipso-ring, meta and para 
respectively). In a previous work, ${ }^{35}$ we dealt with the substituent effect on RAHB distances for substituted o-hydroxybenzaldehyde. It was found that depending on the electronic character of the substituents the RAHB is strengthened or weakened. As can be seen in Scheme 5, EDG and EWG in para an ortho position to each other helps to activate the RAHB. For that reason LM-1 presents a shorter RAHB distance $(1.736 \AA)$ as compared to o-hydroxybenzaldehyde $(1.758 \AA)$, whereas the RAHB in LP-1 is deactivated (1.790 $\AA$ ), as an EWG $(\mathrm{CHO})$ is added to position 4 and an EDG $(\mathrm{OH})$ is attached to position 5. The difference of $0.054 \hat{A}$ in the RAHB bond length of LM-1 and LP-1 reflects the substituent effect. As a result of stronger RAHBs, LM-1 is more stable than its LP-1 isomer by $8.15 \mathrm{kcal}$ $\mathrm{mol}^{-1}$ (see Table 1).

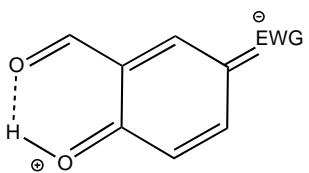

(a)<smiles>O=C1C=CC=C2ONOC=C12</smiles>

(c)<smiles></smiles>

(b) $\ominus_{E W G}$

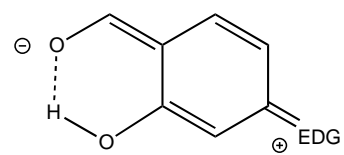

(d)

Scheme 5. The resonance structures responsible for the HB strengthening. EWG in positions 3 and 5 and EDG in positions 4 and 6 activate the RAHB (see Figure 1 for numbering). For further details see ref. 35.

When the second quasi-ring is added at the ortho positions, we can get three different kinked conformers, named KP-1a, KP-1b, and KM-1 (KP-1a for OH and $\mathbf{K P - 1 b}$ for HCO in para positions). As shown in Figure 1, RAHBs in KP-1a and KM1 become shortened as compared to the o-hydroxybenzaldehyde reference, whereas KP-1b remains almost unaffected. KM-1 is activated because of the presence of an EWG in position 3 (Scheme $5 b$ ) and an EDG in position 4 (Scheme 5d). KP-1a and KP-1b are not activated by substituents when forming the RAHB, as EDG and EWG of the quasi-rings are, respectively, in positions 5 and 6 in KP- 
1a and 3 and 4 in KP-1b. The slight shortening in KP-1a can be explained because of a $\mathrm{H} \cdots \mathrm{H}$ repulsion interaction. Indeed, when one of the $\mathrm{HCO}$ groups in KP-1a is rotated by $90^{\circ}$ through the $\mathrm{C}_{\text {ring }}-\mathrm{C}_{\mathrm{co}}$ bond to place it perpendicular to the $6-\mathrm{MR}$ in such a way that both the RAHB and the $\mathrm{H} \cdots \mathrm{H}$ repulsion interaction are broken, the RAHB that remains increases its bond length by $0.048 \AA$. Moreover, we attributed the small deactivating effect in KP-1b to the $O \cdots O$ lone pair repulsions between the two $\mathrm{OH}$ groups. Table 2 shows that $\mathbf{K M}-\mathbf{1}$ is much more stable than $\mathbf{K P}-\mathbf{1 a}$ and KP-1b (10.86 kcal mol-1 and $9.09 \mathrm{kcal} \mathrm{mol}^{-1}$ respectively). The local dipole-dipole repulsions together with $\mathrm{H} \cdots \mathrm{H}$ and $\mathrm{O} \cdots \mathrm{O}$ repulsions are the main causes for the destabilization of $\mathbf{K P}-\mathbf{1 a}$ and $\mathbf{K P - 1 b}$ with respect to $\mathbf{K M - 1}$. It is worth mentioning that a weak HB (2.423 $\AA$ ) between aldehyde group and oxygen of the hydroxyl group may also slightly favor the stabilization of the KM-1 isomer.

If we now focus on aromaticities of the ipso-rings, which are collected in Table 1 and Table 2 for linear and kinked compounds respectively, we can state that there is not a direct relation between the aromaticity of the ipso-ring and the HB distances, although the systems with stronger RAHBs are the ones having the less aromatic ipso-rings. Thus, from these data one can observe that different effects are the responsible of the RAHB length in the quasi-ring, being not always very easy to be predicted the main factor ruling the change in the HB distance. To decrease substituent effects, one can increase the distance between them by adding extra benzene rings and building PAHs with two o-hydroxyaldehyde substituents with two different topologies, namely, linear (named LM-n and LP-n, where $\mathbf{n}$ is the number of benzene rings) and kinked (KM-na, KM-nb, and KP-n following the nomenclature of Figure 1 and being $n$ the number of benzene rings). All these new compounds resemble [ $n$ ]acenes (linear) and [ $n$ ]phenanthrenes (kinked). It is worth mentioning that the maximum number of added benzene rings in this work is 5 , as it was found that for [ $n$ ] acenes with larger $n$ values, open-shell biradical singlet state can be more stable that closed-shell singlet state. ${ }^{40,41}$ 
Figure 2 depicts the linear structures studied with the corresponding RAHB distances and the labels of the 6-MRs. Aromaticity of the different 6-MRs and quasi-rings are collected in Table 1. Let's start adding one ring to both LP-1 and LM-1 systems, so giving LP-2 and LM-2. The addition of a first ring brings to a reduction of the strength of RAHBs that have longer HB distances. There are two electronic effects that explain the weakening of RAHBs in LP-2 and LM-2. First, the substituent effect decreases as the number of benzene rings increases. This is especially important in the case of LP-2 for which it is not possible to find monoionic resonance structures that favor the RAHB. For LM-2, the monoionic resonance structure in Scheme 6 (and its symmetric counterpart not depicted) indicates that substituent effects favor both RAHBs as shown already by Krygowski et al. ${ }^{42}$ but the effect is not as important as in LM-1 due to the increased distance between substituents.
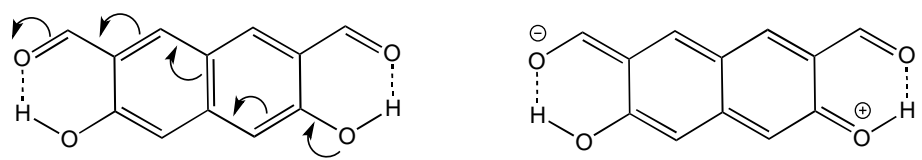

\section{LM-2}

Scheme 6. Substituted naphthalene resonance structure responsible for the HB strengthening in LM-2.

Second, the aromaticity of the 6-MRs when going from LP-1 or LM-1 to LP-2 or LM-2 compounds decreases and the $\pi$-density is partially localized in the C2-C3, C4-C5, and $\mathrm{C} 6-\mathrm{C} 1$ bonds (see Figure 1 for labels). Then, the $\mathrm{C} 1-\mathrm{C} 2$ bond having the two substituents has less $\pi$-electrons available for resonance assistance (C1C2 bond distances are $1.425 \AA$ for LM-1 and $1.435 \AA$ for LM-2) and, consequently, the HB is weakened. The same trend is also followed when adding more benzene rings, showing a convergence of hydrogen bond distances and aromaticities for $n$ $>3$. Linear PAHs present a migrating $\pi$-sextet (see Scheme 4 ), with similar aromaticities for the different rings. The convergence between LP and LM aromaticities can be seen in Table 1. LM-1 and LP-1 start with very different 
aromaticities ( 0.0563 and 0.0817 respectively) and they converge to similar values (0.0435 and 0.0442 for ipso-rings in LM-5 and LP-5 respectively).

Table 1. PDI values (in e) for the benzene rings and quasi-rings (PDlQ) of the linear systems. $\Delta \mathrm{E}_{\text {rel }}$ is the relative energy between $\mathbf{L M n}$ and $\mathbf{L P n}$ isomers $\left(\mathrm{kcal} \mathrm{mol}^{-1}\right)$. See Figure 2 for labels of the rings.

\begin{tabular}{|c|c|c|c|c|c|c|}
\hline \multirow{4}{*}{$\begin{array}{c}\text { Systems } \\
\text { LM-1 } \\
\text { LP-1 }\end{array}$} & \multicolumn{3}{|c|}{ PDI } & \multicolumn{2}{|c|}{$\mathrm{PDI}_{\mathrm{Q}}$} & \multirow[t]{2}{*}{$\Delta \mathrm{E}_{\mathrm{rel}}$} \\
\hline & $\bar{A}$ & B & C & $A^{\prime}$ & $\mathrm{B}^{\prime}$ & \\
\hline & 0.0563 & & & 0.0107 & 0.0107 & - \\
\hline & 0.0817 & & & 0.0134 & 0.0134 & 8.15 \\
\hline LM-2 & 0.0553 & 0.0553 & & 0.0080 & 0.0080 & - \\
\hline LP-2 & 0.0649 & 0.0649 & & 0.0086 & 0.0086 & 3.20 \\
\hline LM-3 & 0.0503 & 0.0616 & 0.0503 & 0.0064 & 0.0064 & - \\
\hline LP-3 & 0.0540 & 0.0670 & 0.0540 & 0.0066 & 0.0066 & 1.37 \\
\hline LM-4 & 0.0462 & 0.0599 & 0.0599 & 0.0055 & 0.0055 & - \\
\hline LP-4 & 0.0477 & 0.0619 & 0.0619 & 0.0056 & 0.0056 & 0.62 \\
\hline LM-5 & 0.0435 & 0.0577 & 0.0631 & 0.0050 & 0.0050 & - \\
\hline LP-5 & 0.0442 & 0.0588 & 0.0648 & 0.0050 & 0.0050 & 0.28 \\
\hline
\end{tabular}

Table 1 also collects the relative energy between $\mathbf{L M}$ and $\mathbf{L P}$ isomers, with $\mathbf{L M}$ being always the most stable one. When only one benzene ring is separating both quasi-rings (LP-1 and LM-1) the energy difference is quite large $\left(8.15 \mathrm{kcal} \mathrm{mol}^{-1}\right)$. When the number of 6-MR is increasing, the relative stability of LP-n with respect to $\mathbf{L M}-\mathbf{n}$ decreases to $0.28 \mathrm{kcal} \mathrm{mol}^{-1}$, the two isomers being almost degenerated for $n=5$. Not only relative energies and aromaticities converge with the increase of $n$, but also HB lengths as shown in Figure 2. Then, despite the substituent effect favors the RAHB in LM-n systems and disfavors it in LP-n species, there is a convergence in the relative energies, aromaticities, and HB lengths of this two series of compounds, indicating that substituent effects becomes negligible for systems with $n>3$. 

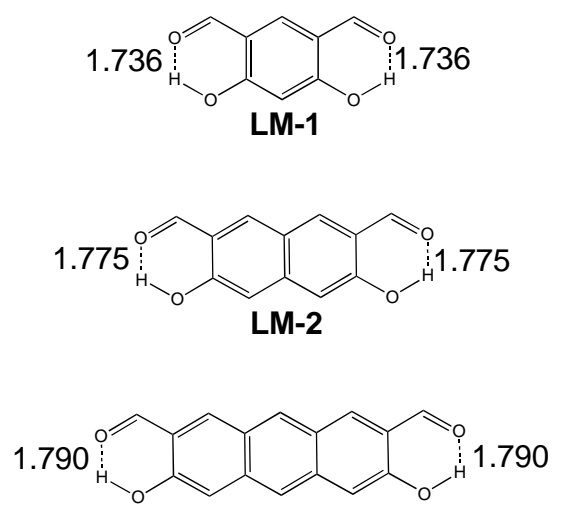

LM-3

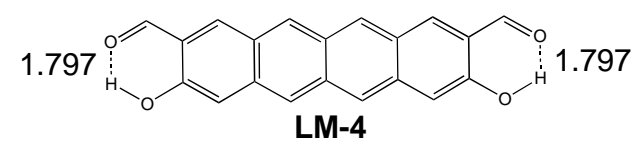

LM-4

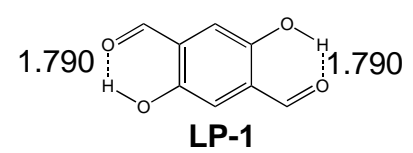

LP-1
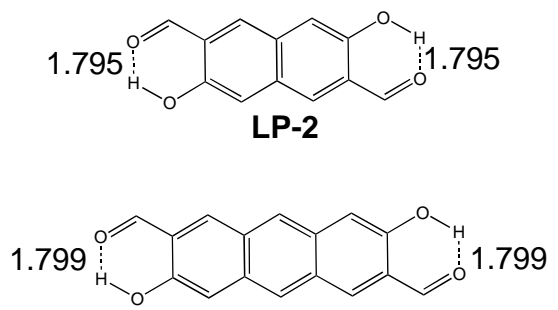

LP-3

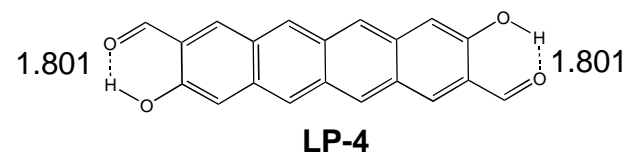

LP-4

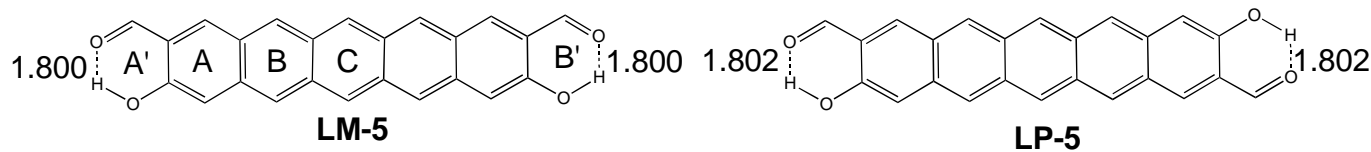

Figure 2. [n]Acene-like isomers $(n=1-5)$. Bond lengths in $\AA$.

Now let's focus on kinked systems, which are built from KP-1a, KP-1b, and KM-1, by adding extra 6-MRs to generate the different [ $n$ ]phenacene-like compounds depicted in Figure 3. KM has been used to name systems with the same substituent building the quasi-rings located in "meta" position one with respect the other, i.e., not connected by a straight axis containing the two most separated $\mathrm{C}$ atoms. Similar to the linear set, we build kinked PAHs up to five benzene rings. The main difference with respect to linear conformers is that, for each system, we can have two different quasi-rings with RAHB distance. When one ring is added, the hydrogen bond distances increase or decrease depending on aromaticity of the ipso-ring and the substituent effect. However, again, the substituent effect is partially neutralized due to the existence of different $\pi$-delocalization paths. As before, the RAHB distances tend to converge while increasing the number of benzene rings. Relative energies between kinked and linear (LM-n as reference) are collected in Table 2, $\Delta \mathrm{EK}_{\mathrm{K} \text {-L. }}$ As the number of kinked rings increase, they are much more stable than the linear isomers, in agreement with previous studies of 
Poater et al. ${ }^{38}$ It is worth noting that the relative energy of the different kinked isomers for $\mathrm{n} \geq 2$ depends mainly on the number of $\mathrm{H} \cdots \mathrm{H}$ repulsion interactions (2HRls) between the $\mathrm{H}$ atoms located in the bay regions of the kinked species. The higher the number of $2 \mathrm{HRI}$, the lower the stability of the isomer. For instance, $\mathbf{K M}-\mathbf{2 b}$ (two 2HRI) is less stable than KP-2 (one 2HRI) and this in turn is less stable than KM-2a (no 2HRI). Or the order of stability for $n=3$ is KP-3a (three $3 \mathrm{HRI}$ ) < KM-3 (two 2HRI) < KP-3b (one 2HRI). And the same for $n=4$ and 5 (see Figure $4)$.

Table 2. PDI values (in e) for the benzene rings and quasi-rings ( $P D l_{Q}$ ) of the kinked systems. $E_{(K-L)}$ is the relative energy to LM-n while $E_{\text {rel }}$ is the relative energy between KM-n and KP-n isomers $\left(\mathrm{kcal} \mathrm{mol}^{-1}\right)$

\begin{tabular}{|c|c|c|c|c|c|c|c|c|c|}
\hline \multirow{4}{*}{$\begin{array}{l}\text { Systems } \\
\text { KM-1 } \\
\text { KP-1a } \\
\text { KP-1b }\end{array}$} & \multicolumn{5}{|c|}{ PDI } & \multicolumn{2}{|c|}{$\mathrm{PDI}_{\mathrm{Q}}$} & \multirow{2}{*}{$\begin{array}{r}\Delta \mathrm{E}_{\mathrm{K}-\mathrm{L}^{\mathrm{a}}} \\
0.00\end{array}$} & \multirow[t]{2}{*}{$\Delta \mathrm{E}_{\mathrm{rel}}^{\mathrm{b}}$} \\
\hline & A & B & C & D & $E$ & $\mathbf{A}^{\prime}$ & B' $^{\prime}$ & & \\
\hline & $\begin{array}{l}0.0506 \\
0.0668\end{array}$ & & & & & $\begin{array}{l}0.0149 \\
0.0202\end{array}$ & $\begin{array}{l}0.0138 \\
0.0202\end{array}$ & $\begin{array}{r}0.00 \\
10.86\end{array}$ & 10.86 \\
\hline & 0.0704 & & & & & 0.0170 & 0.0170 & 9.09 & 9.09 \\
\hline KM-2a & 0.0609 & 0.0609 & & & & 0.0192 & 0.0192 & -3.56 & \\
\hline KM-2b & 0.0592 & 0.0592 & & & & 0.0195 & 0.0195 & 2.75 & 6.30 \\
\hline KP-2 & 0.0539 & 0.0575 & & & & 0.0179 & 0.0178 & -3.13 & 0.42 \\
\hline KM-3 & 0.0611 & 0.0509 & 0.0599 & & & 0.0179 & 0.0179 & -9.15 & 2.21 \\
\hline KP-3a & 0.0608 & 0.0540 & 0.0608 & & & 0.0187 & 0.0187 & -5.00 & 6.36 \\
\hline KP-3b & 0.0632 & 0.0519 & 0.0632 & & & 0.0182 & 0.0182 & -11.36 & \\
\hline KM-4a & 0.0613 & 0.0551 & 0.0551 & 0.0613 & & 0.0840 & 0.0184 & -19.39 & \\
\hline KM-4b & 0.0600 & 0.0557 & 0.0557 & 0.0600 & & 0.0186 & 0.0186 & -13.02 & 6.37 \\
\hline KP-4 & 0.0591 & 0.0563 & 0.0542 & 0.0609 & & 0.0184 & 0.0182 & -16.53 & 2.86 \\
\hline KM-5 & 0.0610 & 0.0540 & 0.0585 & 0.0546 & 0.0597 & 0.0182 & 0.0184 & -24.79 & 3.00 \\
\hline KP-5a & 0.0599 & 0.0553 & 0.0589 & 0.0553 & 0.0599 & 0.0185 & 0.0185 & -21.47 & 6.33 \\
\hline KP-5b & 0.0614 & 0.0535 & 0.0600 & 0.0535 & 0.0614 & 0.0183 & 0.0183 & -27.79 & \\
\hline
\end{tabular}

${ }^{a} \mathbf{L M}-\mathbf{n}$ is the reference system for energy difference

b The most stable systems between KM-n and KP-n is taken as the reference system 


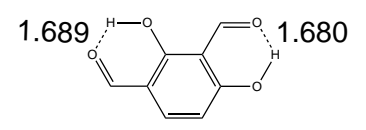

KM-1

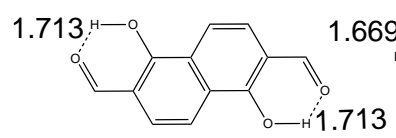

KM-2a
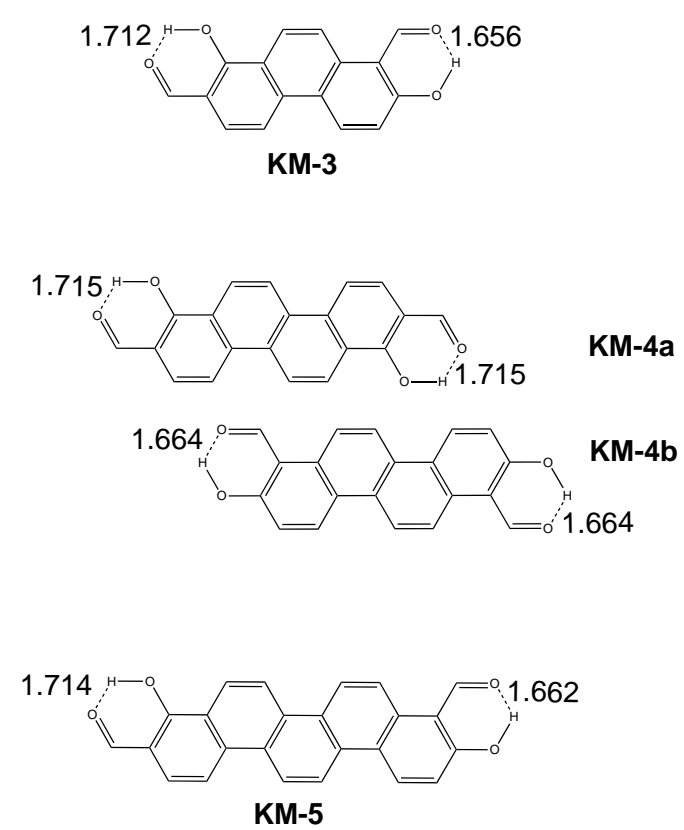

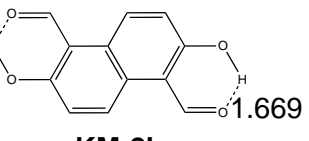

KM-2b

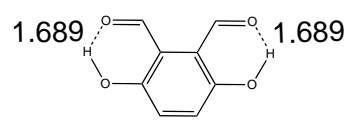

KP-1a

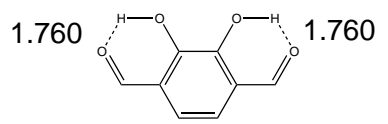

KP-1b

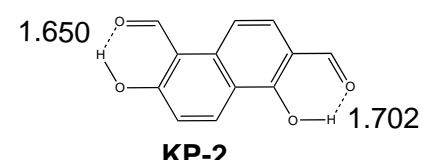

KP-2
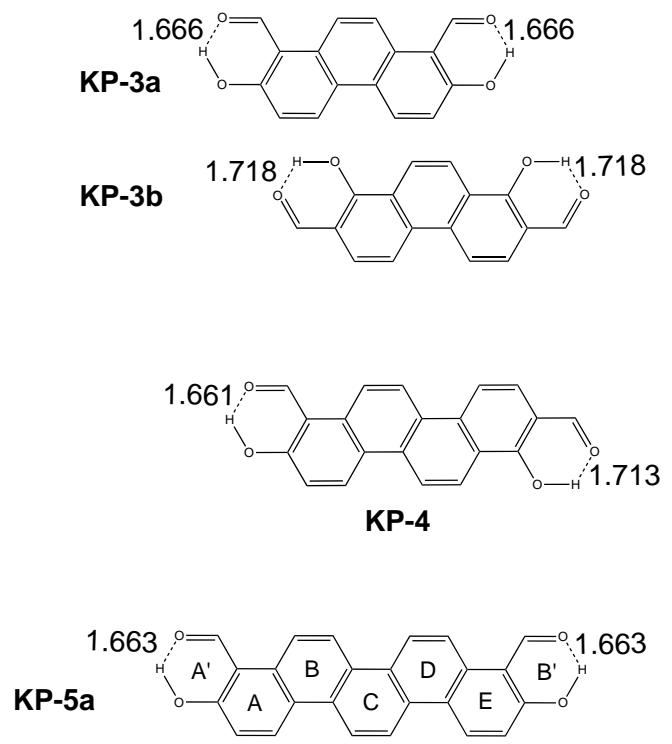

KP-5b

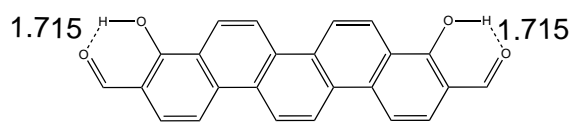

Figure 3. [n]Phenacene-like isomers $(n=1-5)$. Bond lengths in $\AA$.

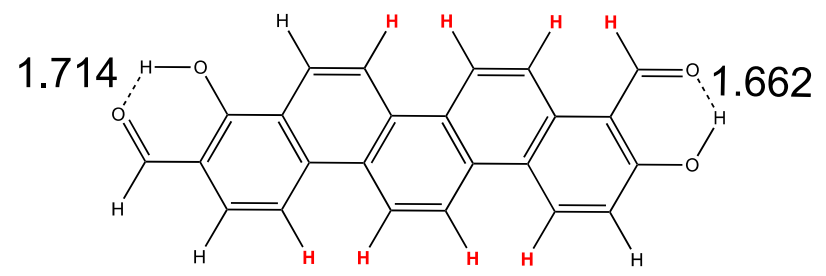

KM-5

Figure 4. The four $\mathrm{H} \cdots \mathrm{H}$ repulsion interactions in $\mathrm{KM}-5$ molecule indicated in red color. Bond lengths in $\AA$.

Previous studies ${ }^{36}$ reported that, in [n]phenacenes, the aromaticity is larger in the external rings than in the central ones, while in [n]acenes external rings are slightly 
aromatic than inner ones. For our systems, this fact should be translated in more aromatic quasi-rings for $\mathbf{K P}$ and $\mathbf{K M}$ compounds as compared to LP and LM systems. Indeed, this is what is found and, for instance, PDlQ is 0.005 e in LM-5 and four time higher $(0.0182 \mathrm{e})$ in KM-5. This is in agreement with the HB strength of quasi-rings, being stronger, with shorter RAHB distance, in kinked compounds (with larger aromaticity for the quasi-ring) than in their linear counterparts. In addition, kinked PAHs have an alternation of the aromaticity from the external to the central rings. This alternation can easily be predicted by Clar's aromatic $\pi$ sextet model (Scheme 4).

When adding one more ring to KP-1a to build KP-2, we can see that quasi-rings in KP-2 have quite different HB distances (1.650 $\AA$ and $1.702 \AA$ for A' and B' respectively) but similar aromaticities (0.0179 and $0.0178 \mathrm{e})$. This change on the $\mathrm{HB}$ distances is neither related to the aromaticity of the ipso-ring $(0.0539$ e for $\mathbf{A}$ and 0.0575 e for $\mathbf{B})$ nor to the double bond character of the shared C1-C2 (1.403 $\AA$ for $\mathbf{A}^{\prime}$ and $1.395 \AA$ for $\mathbf{B}^{\prime}$ ring). The difference in these distances can be understood if we take into account that quasi-ring $\mathbf{A}^{\prime}$ is affected by a $2 \mathrm{HRI}$ that "artificially" makes the RAHB shorter, whereas quasi-ring B' is free of this type of interactions. The same behavior is found for the rest of isomers (e.g. $1.713 \AA$ for KM-2a with no $2 \mathrm{HRI}$ and $1.669 \AA$ for $\mathbf{K M}-2 \mathrm{~b}$ with two $2 \mathrm{HRI}$ ). To validate the decrease of the $\mathrm{HB}$ distance due to the presence of $2 \mathrm{HRI}$, we have removed one of the hydrogen atoms involved in the repulsion to generate a radical structure without $2 \mathrm{HRI}$. In the case of radical KM-5 generated after removal of a single hydrogen (the one close to the $\mathrm{CHO}$ group), the $\mathrm{HB}$ length increases from 1.662 $\AA$ to $1.719 \AA$, which is very close to the HB length in the second quasi-ring (1.715 $\AA$ ) i.e. the one without $H \cdots H$ repulsion. So, indeed, the repulsion seems to be responsible for shortening the HB distance for about $0.06 \AA$. Moreover, in the case of KP-5a, removal of the repulsion between two $\mathrm{H}$ on both sites (the ones on phenyl rings close to the quasi-ring) leads to the increase of HB length from 1.663 $\AA$ in KP-5a (with $\mathrm{H} \cdots \mathrm{H}$ repulsion) to $1.720 \AA$ in the biradical system with no $\mathrm{H} \cdot \cdot \mathrm{H}$ 
repulsion affecting the RAHB. Finally, let us note that at variance with the linear species, the RAHB distances converge already for $n=2$.

From our results, we can see that RAHB distance can be tuned by adding a set of 6-MRs between quasi-rings. When a linear chain is built, the RAHB distance increases, whereas for kinked chains the lenght of RAHB remains quite constant. Clar's aromatic $\pi$-sextet model extended to quasi-rings helps to explain the different RAHB lenght and aromaticity of the ipso and quasi-rings in linear and kinked compounds. Nevertheless, the two different RAHB lengths found in some kinked systems and the fact that the most stable isomers have, in some cases, larger RAHB distances cannot be explained with the Clar model. To explain these results one needs to resort to $\mathrm{H} \cdots \mathrm{H}$ repulsive interactions.

\section{On the way from linear to kinked structures}

As said in the previous section, the general trend of the bond lengths in RAHBs of kinked and linear PAHs can be explained using the extended Clar's model that relates aromaticity of the ipso- and quasi-rings with the RAHB distance. To get deeper insight into the different behavior of linear LM-5 and kinked KP-5a species, we transformed LM-5 compound to KP-5a by kinking one by one the different rings, so going through LM-5' (one kink), KP-5' (two kinks) and finally KP-5a (three kinks). Figure 5 schematically draws the four different compounds that brings to the kinked KP-5a from linear LP-5. Starting from one kink in right side (LM-5'), there is an important decrease of the HB distance (from $1.800 \AA$ to $1.665 \AA$ ), while the HB distance of the quasi-ring that keeps in a linear topology remains almost unchanged. As it can be seen in Table 3, this change in the topology goes together with important change in the aromaticity of the different rings. Following expectations from Clar's model, there is a decrease of aromaticity in the $\mathbf{D}$ ring (phenanthrene-like topology) in favor of an increase in $\mathbf{E}$ ring. As pointed out before, the kinked quasi-ring (B') with shorter HB distance becomes much more aromatic. 
Table 3. PDI values (in e) of the benzene rings and quasi-rings ( $P D l_{Q}$ ) of the systems studied along the change from linear LM-5 to kinked KP-5a structure. Relative energies referred to LM-5 $\left(\mathrm{kcal} \mathrm{mol}^{-1}\right)$

\begin{tabular}{|c|c|c|c|c|c|c|c|c|}
\hline \multirow{3}{*}{$\begin{array}{l}\text { System } \\
\text { LM-5 }\end{array}$} & \multicolumn{5}{|c|}{ PDI } & \multicolumn{2}{|c|}{$\mathrm{PDI}_{\mathrm{Q}}$} & \multirow[t]{2}{*}{$\mathrm{E}_{\text {rel }}$} \\
\hline & $\mathbf{A}$ & B & C & D & $E$ & $A^{\prime}$ & $B^{\prime}$ & \\
\hline & 0.0435 & 0.0577 & 0.0631 & 0.0577 & 0.0435 & 0.0050 & 0.0050 & 0.00 \\
\hline LM-5' & 0.0495 & 0.0634 & 0.0613 & 0.0309 & 0.0665 & 0.0060 & 0.0170 & -11.42 \\
\hline KP-5' & 0.0654 & 0.0387 & 0.0731 & 0.0401 & 0.0638 & 0.0172 & 0.0176 & -23.44 \\
\hline KP-5a & 0.0599 & 0.0553 & 0.0589 & 0.0553 & 0.0599 & 0.0185 & 0.0185 & -21.47 \\
\hline
\end{tabular}
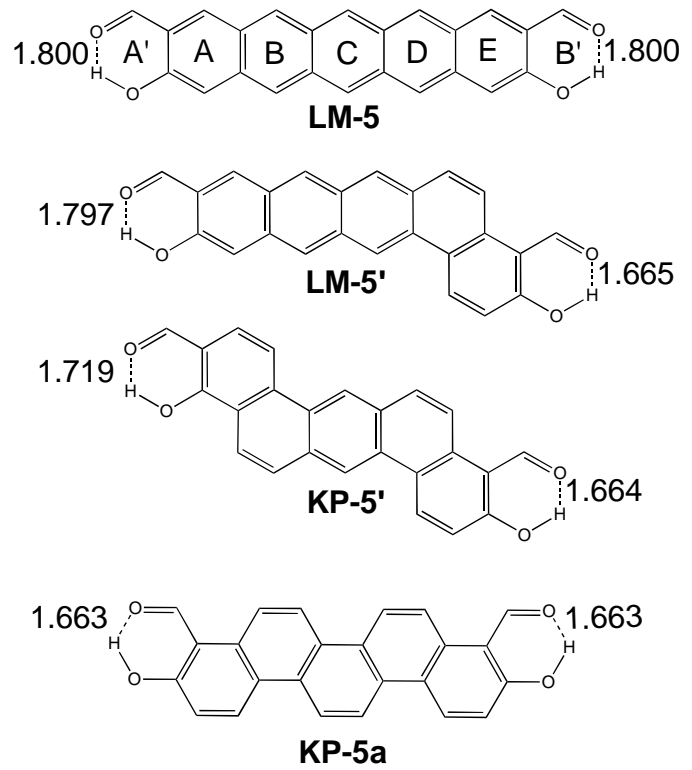

Figure 5. From linear to kinked PAHs. Bond lengths in $\AA$.

Now we can proceed kinking the other side of the chain, obtaining the KP-5' compound. We observed a decrease of the HB distance to $1.719 \AA$, but not as large as in the previous kink (1.665 $\AA$ ). The difference again the presence of a $2 \mathrm{HRI}$ in the first kink and not in the second one. As seen in the previous kink, there is an important lost of aromaticity in ring $\mathbf{B}$ and an increase in rings $\mathbf{A}$ and $\mathbf{A}^{\prime}$. To get the total kinked compound KP-5a, we proceed to twist the central part of the chain. Aromaticity of the central rings becomes more similar to each other, with larger values for A, C, and E, as it was expected from Clar's model. Quasi-ring aromaticities present no important changes, as their kinked topology do not change when kinking the central part of the chain. The C1-C2 bonds bearing the 
substituents in the two outer aromatic rings changes from $1.451 \AA$ in LM-5 to 1.399 $\AA$ in KP-5a, thus indicating an increase in the double character of this bond when going from linear to kinked structures. As before, the aromaticity of the ipso- and quasi-rings increases when going from the linear to the kinked topology.

Finally, Table 3 also collects the relative energies of the different conformers with respect to the linear one (LM-5). As it was expected, when kinking only one site, there is an important stabilization $\left(-11.42 \mathrm{kcal} \mathrm{mol}^{-1}\right)$. This agrees with previous work showing that phenanthrene is more stable than anthracene because of better $\pi$-interactions. ${ }^{39}$ When the other site of the chain is kinked, the stabilization energy increases up to $-23.44 \mathrm{kcal} \mathrm{mol}^{-1}$, so reinforcing the stabilization because of phenanthrene-like topology being more stable. In the last conformer, where all the chain is kinked, the relative energy decreases to $-21.47 \mathrm{kcal} \mathrm{mol}^{-1}$ but because there is an increase of hydrogen repulsion through the whole chain, five $2 \mathrm{HRI}$ in KP-5a to be compared to three 2HRI for KP-5'.

\section{Triphenylene-like RAHB}

Let us move one step further and consider a set of compounds with two quasirings that resemble triphenylene (see Scheme 7). Starting from KP-1a, KP-1b, and $\mathbf{K M}-1$, one can add successive benzene rings in the ipso-ring keeping the original two quasi-rings to generate the compounds depicted in Figure 6 named KP-Lna, $\mathrm{KP}$-Lnb, and KM-Ln, with $n$ being the number of benzene rings.

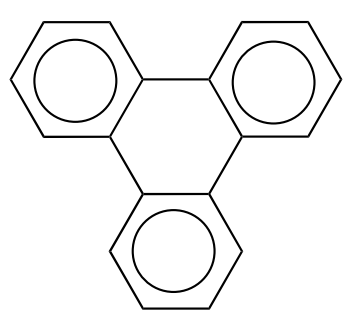

(a)

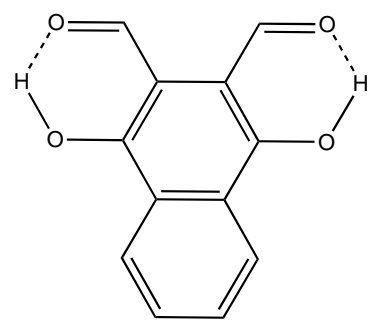

(b)

Scheme 7. (a) Clar's structure for triphenylene (b) triphenylene-like double-headed quasi-ring compound 


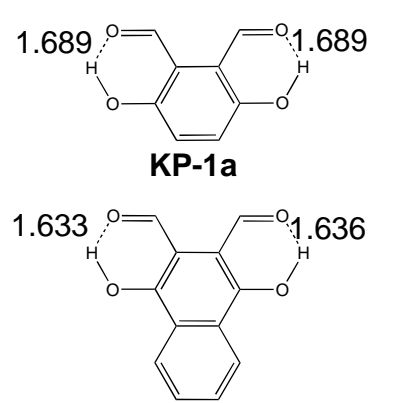

KP-L2a

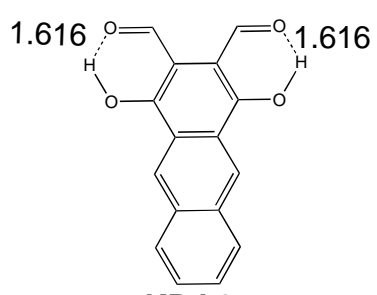

KP-L3a

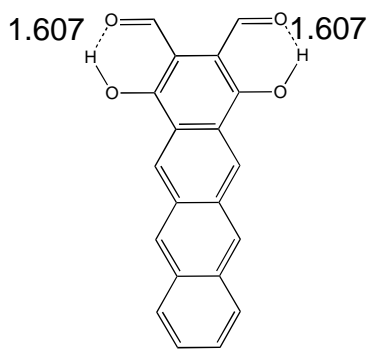

KP-L4a
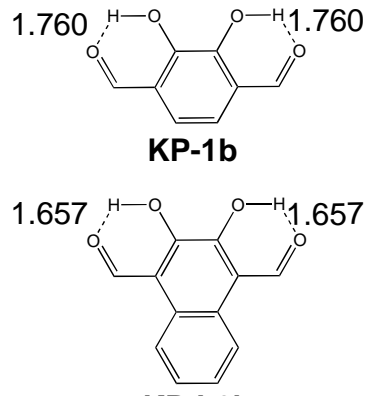

KP-L2b
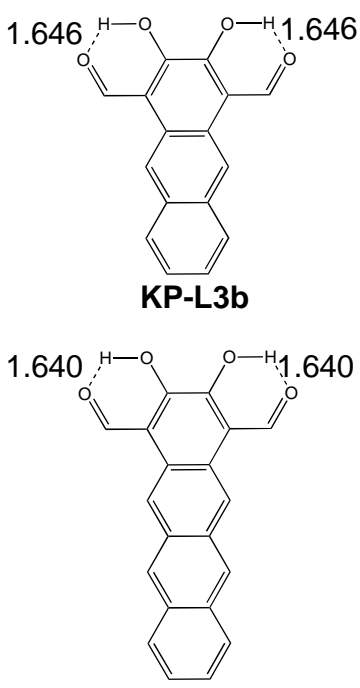

KP-L4b
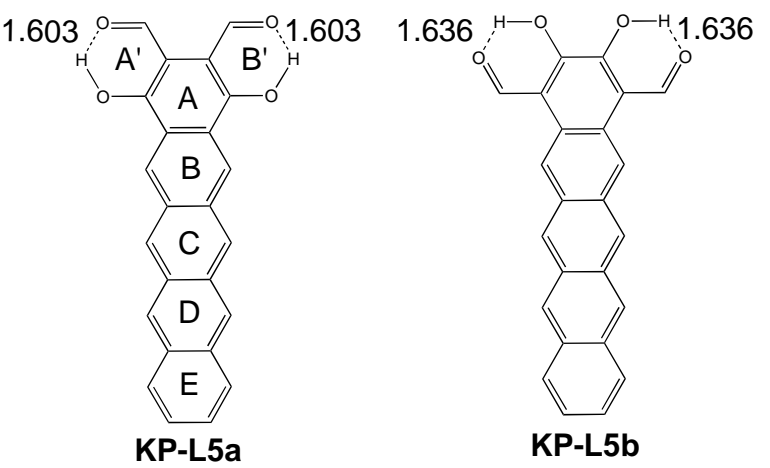
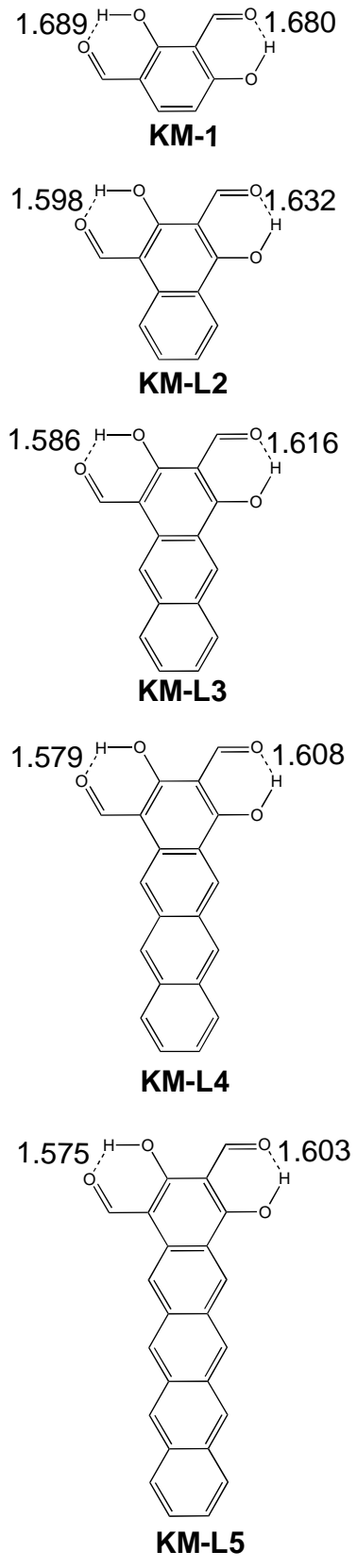

Figure 6. Triphenylene-like studied structures. Bond lengths in $\AA$.

For systems with triphenylene-like structures, substituent effects on the RAHB of the quasi-rings remain the same in each series of compounds (for each column in Figure 6). After adding one ring (KP-L2a, KP-L2b, and KM-L2) an important shortening of the RAHB distances are observed. According to Clar's $\pi$-sextet model (Scheme 6), the central ring in triphenylene is an "empty ring" while the 
outer rings are the most aromatic ones. Analyzing data collected in Table 4, one can observe the ipso-rings have much smaller aromaticities than the added benzene ring (e.g. for KP-L2a 0.0344 e for ring A and 0.0860 e for ring B). For these compounds it is expected that aromaticity of all external ring being large, so quasi-ring also increase their aromaticity when adding a benzene ring (from 0.0170 e for KP-1b to 0.0217 e for KP-L2b).

Table 4. PDI values (in e) of benzene rings and quasi-rings (PDlQ) of the different triphenylenelike systems. Relative energies respect to KM- $\mathrm{Ln}\left(\mathrm{kcal} \mathrm{mol}^{-1}\right)$.

\begin{tabular}{|c|c|c|c|c|c|c|c|c|}
\hline \multirow[b]{2}{*}{$\begin{array}{l}\text { Systems } \\
\text { KP-1a }\end{array}$} & \multicolumn{5}{|c|}{ PDI } & \multicolumn{2}{|c|}{$\mathbf{P D l}_{\mathbf{Q}}$} & \multirow{2}{*}{$\begin{array}{c}E_{\text {rel }} \\
10.86\end{array}$} \\
\hline & $\begin{array}{c}\mathbf{A} \\
0.0668\end{array}$ & B & C & D & $E$ & $\begin{array}{c}\mathbf{A}^{\prime} \\
0.0202\end{array}$ & $\begin{array}{c}\mathbf{B}^{\prime} \\
0.0202\end{array}$ & \\
\hline KP-1b & 0.0704 & & & & & 0.0170 & 0.0170 & 9.09 \\
\hline $\mathrm{KM}-1$ & 0.0586 & & & & & 0.0149 & 0.0138 & \\
\hline KP-L2a & 0.0344 & 0.0860 & & & & 0.0242 & 0.0242 & 6.22 \\
\hline KP-L2b & 0.0383 & 0.0870 & & & & 0.0217 & 0.0217 & 12.31 \\
\hline KM-L2 & 0.0329 & 0.0843 & & & & 0.0197 & 0.0175 & \\
\hline KP-L3a & 0.0243 & 0.0682 & 0.0699 & & & 0.0259 & 0.0259 & 5.68 \\
\hline KP-L3b & 0.0287 & 0.0703 & 0.0699 & & & 0.0230 & 0.0230 & 12.49 \\
\hline KM-L3 & 0.0238 & 0.0689 & 0.0691 & & & 0.0212 & 0.0183 & \\
\hline KP-L4a & 0.0206 & 0.0590 & 0.0660 & 0.0613 & & 0.0267 & 0.0267 & 5.47 \\
\hline KP-L4b & 0.0249 & 0.0617 & 0.0670 & 0.0609 & & 0.0237 & 0.0237 & 12.61 \\
\hline KM-L4 & 0.0201 & 0.0595 & 0.0667 & 0.0610 & & 0.0218 & 0.0191 & \\
\hline KP-L5a & 0.0189 & 0.0552 & 0.0633 & 0.0628 & 0.0564 & 0.0268 & 0.0268 & 5.47 \\
\hline KP-L5b & 0.0227 & 0.0563 & 0.0658 & 0.0627 & 0.0561 & 0.0239 & 0.0239 & 12.72 \\
\hline KM-L5 & 0.0181 & 0.0545 & 0.0635 & 0.0634 & 0.0563 & 0.0221 & 0.0193 & \\
\hline
\end{tabular}

The aromaticity of the ipso-ring can be tuned by adding extra 6-MRs to it, following a linear topology. As it was commented before, for [ $n$ ]acenes with migrating $\pi$ sextet, the aromaticity of all rings decreases while increasing the size of the chain. Keeping this idea in mind, results show that the aromaticity of the ipso-ring, which has from the very beginning a low aromaticity because it resembles to the central ring of triphenylene, decreases with the increase in the number of 6-MRs attached to the ipso-ring. Because of that, the $\mathrm{C}=\mathrm{C}$ bond in the ring junction between the ipso-ring and the quasi-ring becomes more localized for larger systems. The increase in the double character of this bond strengthens the RAHB, which becomes very short for KM-L5 (going from $1.689 \AA$ in KM-1 to $1.575 \AA$ in KM-L5). 
As the quasi-ring has more available its $\pi$-electron pair for larger systems, its aromaticity increases going from, for instance, 0.0149 e for ring A' of KM-1 to 0.0221 e for KM-L5. Indeed, for KM-L5, the quasi-ring is more aromatic than the ipso-ring with a PDI of $0.0181 \mathrm{e}$. Another important issue different from the previous linear and kinked systems studied is that convergence is not fully achieved for $n=5$, neither on the RAHB distance nor on the aromaticity of the RAHB quasi-ring.

As it was pointed out before, substituent effect are not changing while enlarging the chain for each series, so we can analyze the relation between aromaticities of the ipso-ring and the quasi-ring and the HB distances. The linear representation for the different parameters can be found in the SI for each quasi-ring. There are four different quasi-rings to take into account; one for KP-Lna, system with one 2HRI, one for KP-Lnb, species with two 2HRI, and two for KM-Ln, with one 2HRI and one weak HB, all of them with different HB distances. Figure S1 in SI shows a very good linear relationship between the aromaticity of the quasi-ring and the ipso-ring for the four series of different RAHB (average $R^{2}$ of 0.999 ); when the aromaticity of the ipso-ring decreases, that of the quasi-ring increases. Previous works ${ }^{33,34}$ reported that an increase of the PDI in the quasi-ring reinforces the RAHB, and the HB distance is shortened. With this idea in mind, we expect to find four linear relationships between both parameters, the aromaticity of the ipso-ring and the HB length. This correlation is fulfilled for A' and B' quasi-rings of KP-1a and quasi-ring $\mathbf{B}^{\prime}$ of $\mathbf{K M}-\mathbf{1}$. For the other two quasi-rings the expected correlation in not followed (Fig S2 in SI). In this case, the calculated HB distances in $\mathbf{A}^{\prime}$ and B' quasi-rings of KP-1b and quasi-ring A' of KM-1 are much longer that expected from the correlation of the series. Comparing these two compounds with the rest of the series, it can be observed that when a second 6-MR is added to KP-1b and KM-1 an extra 2HRI between the $\mathrm{H}$ atom of the $\mathrm{HCO}$ group of the quasi-ring and one of the $\mathrm{H}$ atoms of the added benzene-ring that decreasing the hydrogen bond length. This repulsion is the same in the rest of the series, and therefore, the linear correlation between aromaticity of the quasi-ring, the ipso-ring and the RAHB 
distances is kept, except for the KP-1b and $\mathbf{K M}-\mathbf{1}$ that are not affected by any 2HRI.

The different behavior of KP-1a and KM-1 (ring B') can also be assessed analyzing the relative energy between conformers of the same size (Table 4). These two conformers present a very different stability through $\left(10.86 \mathrm{kcal} \mathrm{mol}^{-1}\right.$ for KP-1a and $9.09 \mathrm{kcal} \mathrm{mol}^{-1}$ for $\mathbf{K P}-\mathbf{1 b}$ both with respect to $\mathrm{KM}-1$ ) when compared to the rest of the series. These relative energies keep constant (approx. 5 and $12 \mathrm{kcal} \mathrm{mol}^{-1}$ respectively) through the series KP-Lna, KP-Lnb and KM-Ln when there are more than one acene-like ring, as the number of $2 \mathrm{HRI}$ remains the same. The relative stability of the three series (KM-Ln being the most stable, followed by KP-Lna and KP-Lnb) is ruled by the 2HRI between quasi-ring and the added benzene ring in KP-Lnb and between both quasi-rings and the added benzene ring in KP-Lna.

In the series of triphenylene-like compounds studied, the substituent effect remains the same. For this reason, in these systems we could assess the importance of $\pi$ delocalization within the ipso and quasi ring (aromaticity). This delocalization assists the HB, shortening it, so reinforcing the concept of resonance-assisted hydrogen bond.

\section{Conclusions}

Double-headed compounds having two intramolecular resonance-assisted hydrogen bonds (RAHB) have been designed with the aim of studying the relation between the RAHB length and the aromaticity of the different ipso-rings. For these purpose, we have built [ $n]$ acenes (linear) and [n]phenancenes (kinked) like compounds with different number of benzene rings. Two different effects are controlling the HB distances, namely, the connectivity between substituents of the ipso-ring and the topology of the benzene chains (being linear or kinked). The first effect has been decreased by adding up to five benzene rings between quasi-rings in [ $n$ ]acenes and $[n]$ phenancenes, so being the topology of the chain the 
responsible of ruling the different $\mathrm{HB}$ strength. The HB lengths observed can be explained in most cases using an extended Clar's aromatic $\pi$-sextet model that incorporates the quasi-rings as if they were rings in benzenoids. In general, shorter $\mathrm{HBs}$ are found for the more aromatic quasi-rings. The existence of $\mathrm{H} \cdots \mathrm{H}$ bond repulsions in some kinked systems explain the exceptions found to this extended Clar's aromatic $\pi$-sextet model.

Finally, in order to assess the relation between aromaticity (based on PDI values) and HB length, triphenylene-like compounds have been studied. In these systems, where the connectivity between substituent is the same, we found good linear correlation between aromaticity of the ipso- and quasi-ring and the RAHB distances, thus reinforcing the Gilli's concept of resonance assisting the hydrogen bond formations in the quasi-ring.

\section{Computational methods}

All molecules considered in this study were calculated using the long-range corrected hybrid CAM-B3LYP level of theory ${ }^{43}$ including the D3 version of Grimme's dispersion with Becke-Johnson damping ${ }^{44}$ to provide a correct description of the distance-dependence of the hydrogen bonding interactions ${ }^{45}$ as well as the inter-ring electron delocalization effects in the condensed benzenoid systems $^{38,46,47,38}$ The standard $6-311++G(d, p)$ basis set was used for all atoms. Vibrational analyses of optimized structures showed that the structures are minima in the potential energy surface. All calculations were carried out with the Gaussian 09 package. ${ }^{48}$ To avoid the numerical-integration accuracy issues ${ }^{49}$ and the basisset dependence problems, ${ }^{50}$ in this study the delocalization indices (DIs) ${ }^{51}$ have been calculated within the representation of natural atomic orbitals (NAO) ${ }^{52}$ available within the $\mathrm{NBO}^{53}$ and JaNPA ${ }^{54}$ program interfaces.

DIs are among the most popular bonding indicators. They are closely related to the covalent bond-order indices ${ }^{55}$ and provide a quantitative information on the 
electron density shared between two atoms/fragments $\mathrm{A}$ and $\mathrm{B}$. Within the NAObased partitioning scheme the delocalization index for one-determinant closedshell systems is congeneric with the quadratic bond-order originally proposed by Wiberg ${ }^{56}$

$$
\delta(\mathrm{A}, \mathrm{B})=2 \sum_{\mu \in \mathrm{A}} \sum_{\nu \in \mathrm{B}}\left|P_{\mu \nu}\right|^{2}
$$

where $P_{\mu v}$ are the elements of the reduced density matrix in the NAO basis. ${ }^{52}$

Based on the finding of Fulton ${ }^{55}$ and Bader ${ }^{57}$ who showed that benzene has larger DIs in para-related atoms than in meta-related ones, the $\mathrm{PDI}^{58}$ index uses the DIs of para-related atoms as a measure of aromaticity for six-membered rings (6-MRs):

$$
P D I=\frac{\delta\left(A_{1}, A_{4}\right)+\delta\left(A_{2}, A_{5}\right)+\delta\left(A_{3}, A_{6}\right)}{3}
$$

For PDI, the larger the index, the greater the aromaticity of the ring. Obviously, this index can only be applied to 6-MRs. We use it to discuss the aromaticity of both the benzene rings (PDI) and the quasi-ring (PDlQ), also known as quasiaromaticity. ${ }^{59}$ It was demonstrated ${ }^{60}$ for simple derivatives of benzene that PDI gives qualitatively the same results as other electronic indices as well as indices like the HOMA ${ }^{61}$ structural index or the NICS magnetic index. ${ }^{49}$ For this reason, in our studies we are using exclusively the PDI index.

\section{Supporting Information}

Linear correlation between PDI for the ipso and quasi-rings (Figure S1) and linear correlation between aromaticity of the ipso-ring and RAHB bond length (Figure S1) for the triphenylene-like compound. Optimized Cartesian coordinates for all compounds considered at the CAM-B3LYP/6-311+G(d,p)+GD3B level of theory are given in a separated file. 


\section{Acknowledgements}

We are grateful for financial support from the Spanish MINECO (CTQ2017-85341-P) and the Catalan DIUE (2017-SGR-39, XRQTC, and ICREA Academia 2014 Award to M.S.) and the FEDER fund (UNGI10-4E-801). D.W.S acknowledges the financial support of the Polish National Science Center within the Sonata Project 2015/17/D/ST4/00558.

\section{References}

(1) Grabowski, S. J. Hydrogen Bonding - New Insights; Springer: The Netherlands, 2006; Vol. 3.

(2) Jeffrey, G. A. An Introduction to Hydrogen Bonding; Oxford University Press: USA, 1997.

(3) Molecular Interactions: From Van Der Waals to Strongly Bound Complexes; Scheiner, S., Ed.; Wiley \& Sons: UK, 1997.

(4) Steiner, T. The Hydrogen Bond in the Solid State. Angew. Chem. Int. Ed. 2002, 41, 48-76.

(5) Desiraju, G. R.; Steiner, T. The Weak Hydrogen Bond in Structural Chemistry and Biology; Oxford University Press: New York, 1999.

(6) Bertolasi, V.; Gilli, P.; Ferretti, V.; Gilli, G. Evidence for ResonanceAssisted Hydrogen Bonding. 2. Intercorrelation between Crystal Structure and Spectroscopic Parameters in Eight Intramolecularly Hydrogen Bonded 1,3-Diaryl1,3-Propanedione Enols. J. Am. Chem. Soc. 1991, 113, 4917-4925.

(7) Bertolasi, V.; Nanni, L.; Gilli, P.; Ferretti, V.; Gilli, G.; Issa, Y. M.; Sherif, O. E. Intramolecular $\mathrm{N}-\mathrm{H} \cdots \mathrm{O}=\mathrm{C}$ Hydrogen-Bonding Assisted by Resonance 
- Intercorrelation between Structural and Spectroscopic Data for 6 Beta-DiketoArylhydrazones Derived from Benzoylacetone or Acetylacetone. New J. Chem. 1994, 18, 251-261.

(8) Gilli, G.; Bellucci, F.; Ferretti, V.; Bertolasi, V. Evidence for Resonance-Assisted Hydrogen Bonding from Crystal-Structure Correlations on the Enol Form of the $\beta$-Diketone Fragment. J. Am. Chem. Soc. 1989, 111, 1023.

(9) Gilli, P.; Bertolasi, V.; Pretto, L.; Ferretti, V.; Gilli, G. Covalent Versus Electrostatic Nature of the Strong Hydrogen Bond: Discrimination among Single, Double, and Asymmetric Single-Well Hydrogen Bonds by Variable-Temperature X-Ray Crystallographic Methods in Beta-Diketone Enol RAHB Systems. J. Am. Chem. Soc. 2004, 126, 3845-3855.

(10) Gora, R. W.; Maj, M.; Grabowski, S. J. Resonance-Assisted Hydrogen Bonds Revisited. Resonance Stabilization vs. Charge Delocalization. Phys. Chem. Chem. Phys. 2013, 15, 2514-2522.

(11) Grosch, A. A.; van der Lubbe, S. C. C.; Fonseca Guerra, C. Nature of Intramolecular Resonance Assisted Hydrogen Bonding in Malonaldehyde and Its Saturated Analogue. J. Phys. Chem. A 2018, 122, 1813-1820.

(12) Guerra, C. F.; Bickelhaupt, F. M.; Snijders, J. G.; Baerends, E. J. The Nature of the Hydrogen Bond in DNA Base Pairs: The Role of Charge Transfer and Resonance Assistance. Chem. Eur. J. 1999, 5, 3581-3594.

(13) Guevara-Vela, J. M.; Romero-Montalvo, E.; Costales, A.; Pendas, A. M.; Rocha-Rinza, T. The Nature of Resonance-Assisted Hydrogen Bonds: A Quantum Chemical Topology Perspective. Phys. Chem. Chem. Phys. 2016, 18, 26383-26390. 
(14) Guevara-Vela, J. M.; Romero-Montalvo, E.; del Río-Lima, A.; Martín Pendás, Á.; Hernández-Rodríguez, M.; Rocha Rinza, T. Hydrogen-Bond Weakening through $\Pi$ Systems: Resonance-Impaired Hydrogen Bonds (RIHB). Chem. Eur. J 2017, 23, 16605-16611.

(15) Guillaumes, L.; Simon, S.; Fonseca Guerra, C. The Role of Aromaticity, Hybridization, Electrostatics, and Covalency in Resonance-Assisted Hydrogen Bonds of Adenine-Thymine (AT) Base Pairs and Their Mimics. ChemistryOpen 2015, 4, 318-327.

(16) Jiang, X.; Zhang, H.; Wu, W.; Mo, Y. A Critical Check for the Role of Resonance in Intramolecular Hydrogen Bonding. Chem. Eur. J 2017, 23, 1688516891.

(17) Kurczab, R.; Mitoraj, M. P.; Michalak, A.; Ziegler, T. Theoretical Analysis of the Resonance Assisted Hydrogen Bond Based on the Combined Extended Transition State Method and Natural Orbitals for Chemical Valence Scheme. J. Phys. Chem. A 2010, 114, 8581-8590.

(18) Lin, X.; Zhang, H.; Jiang, X.; Wu, W.; Mo, Y. The Origin of the NonAdditivity in Resonance-Assisted Hydrogen Bond Systems. J. Phys. Chem. A 2017, 121, 8535-8541.

(19) Mo, Y. Probing the Nature of Hydrogen Bonds in DNA Base Pairs. J. Mol. Model. 2006, 12, 665-672.

(20) Mo, Y.; Bao, P.; Gao, J. Energy Decomposition Analysis Based on a Block-Localized Wavefunction and Multistate Density Functional Theory. Phys. Chem. Chem. Phys. 2011, 13, 6760-6775.

(21) Mohajeri, A. Theoretical Evidences for Resonance-Assisted Hydrogen Bonding. J. Mol. Struct. (Theochem) 2004, 678, 201-205. 
(22) Paul, B. K. Lack of Resonance Assistance in a Classical Intramolecular Hydrogen Bond: An Exploration from Quantum Theory of Atoms-inMolecules Perspective. J. Phys. Org. Chem. 2019, 32, e3999.

(23) Paul, B. K.; Guchhait, N. Geometrical Criteria Versus Quantum Chemical Criteria for Assessment of Intramolecular Hydrogen Bond (IMHB) Interaction: A Computational Comparison into the Effect of Chlorine Substitution on IMHB of Salicylic Acid in Its Lowest Energy Ground State Conformer. Chem. Phys. 2013, 412, 58-67.

(24) Romero-Montalvo, E.; Guevara-Vela, J. M.; Costales, A.; Pendás, Á. M.; Rocha-Rinza, T. Cooperative and Anticooperative Effects in Resonance Assisted Hydrogen Bonds in Merged Structures of Malondialdehyde. Phys. Chem. Chem. Phys. 2017, 19, 97-107.

(25) Sanz, P.; Mó, O.; Yáñez, M.; Elguero, J. Non-Resonance-Assisted Hydrogen Bonding in Hydroxymethylene and Aminomethylene Cyclobutanones and Cyclobutenones and Their Nitrogen Counterparts. ChemPhysChem 2007, 8, 1950-1958.

(26) Sanz, P.; Yáñez, M.; Mó, O. Resonance-Assisted Intramolecular Chalcogen-Chalcogen Interactions? Chem. Eur. J 2003, 9, 4548-4555.

(27) Sobczyk, L.; Grabowski, S. J.; Krygowski, T. M. Interrelation between H-Bond and Pi-Electron Delocalization. Chem. Rev. 2005, 105, 3513-3560.

(28) Houjou, H.; Motoyama, T.; Banno, S.; Yoshikawa, I.; Araki, K. Experimental and Theoretical Studies on Constitutional Isomers of 2,6Dihydroxynaphthalene Carbaldehydes. Effects of Resonance-Assisted Hydrogen Bonding on the Electronic Absorption Spectra. J. Org. Chem. 2009, 74, 520-529. 
(29) Houjou, H.; Shingai, H.; Yagi, K.; Yoshikawa, I.; Araki, K. Mutual Interference between Intramolecular Proton Transfer Sites through the Adjoining П-Conjugated System in Schiff Bases of Double-Headed, Fused Salicylaldehydes. J. Org. Chem. 2013, 78, 9021-9031.

(30) Mahmudov, K. T.; Pombeiro, A. J. L. Resonance-Assisted Hydrogen Bonding as a Driving Force in Synthesis and a Synthon in the Design of Materials. Chem. Eur. J 2016, 22, 16356-16398.

(31) Nguyen, Y. H.; Lampkin, B. J.; Venkatesh, A.; Ellern, A.; Rossini, A. J.; VanVeller, B. Open-Resonance-Assisted Hydrogen Bonds and Competing Quasiaromaticity. J. Org. Chem. 2018.

(32) Dewar, M. J. S. Structure of Stipitatic Acid. Nature 1945, 155, 50.

(33) Palusiak, M.; Simon, S.; Solà, M. Interplay between Intramolecular Resonance-Assisted Hydrogen Bonding and Aromaticity in o-Hydroxyaryl Aldehydes. J. Org. Chem. 2006, 71, 5241-5248.

(34) Palusiak, M.; Simon, S.; Solà, M. Interplay between Intramolecular Resonance-Assisted Hydrogen Bonding and Local Aromaticity. II. 1,3Dihydroxyaryl-2-Aldehydes. J. Org. Chem. 2009, 74, 2059-2066.

(35) Pareras, G.; Palusiak, M.; Duran, M.; Solà, M.; Simon, S. Tuning the Strength of the Resonance-Assisted Hydrogen Bond in o-Hydroxybenzaldehyde by Substitution in the Aromatic Ring. J. Phys. Chem. A 2018, 122, 2279-2287.

(36) Portella, G.; Poater, J.; Bofill, J. M.; Alemany, P.; Solà, M. Local Aromaticity of [n]Acenes, [n]Phenacenes, and [n]Helicenes $(n=1-9)$. J. Org. Chem. 2005, 70, 2509-2521.

(37) Clar, E. The Aromatic Sextet; Wiley: New York, 1972. 
(38) Szczepanik, D. W.; Solà, M.; Krygowski, T. M.; Szatylowicz, H.; Andrzejak, M.; Pawełek, B.; Dominikowska, J.; Kukułka, M.; Dyduch, K. Aromaticity of Acenes: The Model of Migrating $\Pi$-Circuits. Phys. Chem. Chem. Phys. 2018, 20, 13430-13436.

(39) Poater, J.; Visser, R.; Solà, M.; Bickelhaupt, F. M. Polycyclic Benzenoids: Why Kinked Is More Stable Than Straight. J. Org. Chem. 2007, 72, 1134-1142.

(40) Bendikov, M.; Duong, H. M.; Starkey, K.; Houk, K. N.; Carter, E. A.; Wudl, F. Oligoacenes: Theoretical Prediction of Open-Shell Singlet Diradical Ground States. J. Am. Chem. Soc. 2004, 126, 7416-7417.

(41) Yu, D.; Stuyver, T.; Rong, C.; Alonso, M.; Lu, T.; De Proft, F.; Geerlings, P.; Liu, S. Global and Local Aromaticity of Acenes from the InformationTheoretic Approach in Density Functional Reactivity Theory. Phys. Chem. Chem. Phys. 2019, 21, 18195-18210.

(42) M. Krygowski, T.; Palusiak, M.; Goral, A.; E. Zachara-Horeglad, J. Relationship between Substituent Effect and Aromaticity - Part III: Naphthalene as a Transmitting Moiety for Substituent Effect. J. Phys. Org. Chem. 2007, 20, 297306.

(43) Yanai, T.; Tew, D. P.; Handy, N. C. A New Hybrid ExchangeCorrelation Functional Using the Coulomb-Attenuating Method (CAM-B3LYP). Chem. Phys. Lett. 2004, 393, 51-57.

(44) Grimme, S.; Ehrlich, S.; Goerigk, L. Effect of the Damping Function in Dispersion Corrected Density Functional Theory. J. Comput. Chem. 2011, 32, 1456-1465. 
(45) Thanthiriwatte, K. S.; Hohenstein, E. G.; Burns, L. A.; Sherrill, C. D. Assessment of the Performance of DFT and DFT-D Methods for Describing Distance Dependence of Hydrogen-Bonded Interactions. J. Chem. Theory Comput. 2011, 7, 88-96.

(46) Casademont-Reig, I.; Woller, T.; Contreras-García, J.; Alonso, M.; Torrent-Sucarrat, M.; Matito, E. New Electron Delocalization Tools to Describe the Aromaticity in Porphyrinoids. Phys. Chem. Chem. Phys. 2018, 20, 2787-2796.

(47) Szczepanik, D. W.; Andrzejak, M.; Dominikowska, J.; Pawełek, B.; Krygowski, T. M.; Szatylowicz, H.; Solà, M. The Electron Density of Delocalized Bonds (EDDB) Applied for Quantifying Aromaticity. Phys. Chem. Chem. Phys. 2017, 19, 28970-28981.

(48) Frisch, M. J. T., G. W.; Schlegel, H. B.; Scuseria, G. E.; Robb, M. A.; Cheeseman, J. R.; Scalmani, G.; Barone, V.; Mennucci, B.; Petersson, G. A.; Nakatsuji, H.; Caricato, M.; Li, X.; Hratchian, H. P.; Izmaylov, A. F.; Bloino, J.; Zheng, G.; Sonnenberg, J. L.; Hada, M.; Ehara, M.; Toyota, K.; Fukuda, R.; Hasegawa, J.; Ishida, M.; Nakajima, T.; Honda, Y.; Kitao, O.; Nakai, H.; Vreven, T.; Montgomery, Jr., J. A.; Peralta, J. E.; Ogliaro, F.; Bearpark, M.; Heyd, J. J.; Brothers, E.; Kudin, K. N.; Staroverov, V. N.; Kobayashi, R.; Normand, J.; Raghavachari, K.; Rendell, A.; Burant, J. C.; Iyengar, S. S.; Tomasi, J.; Cossi, M.; Rega, N.; Millam, J. M.; Klene, M.; Knox, J. E.; Cross, J. B.; Bakken, V.; Adamo, C.; Jaramillo, J.; Gomperts, R.; Stratmann, R. E.; Yazyev, O.; Austin, A. J.; Cammi, R.; Pomelli, C.; Ochterski, J. W.; Martin, R. L.; Morokuma, K.; Zakrzewski, V. G.; Voth, G. A.; Salvador, P.; Dannenberg, J. J.; Dapprich, S.; Daniels, A. D.; Farkas, Ö.; Foresman, J. B.; Ortiz, J. V.; Cioslowski, J.; Fox, D. J. Gaussian 09, Revision A.02, Gaussian Inc., Wallingford CT, 2009.

(49) Feixas, F.; Matito, E.; Poater, J.; Solà, M. Quantifying Aromaticity with Electron Delocalisation Measures. Chem. Soc. Rev. 2015, 44, 6434-6451. 
(50) Jabłoński, M.; Palusiak, M. Basis Set and Method Dependence in Quantum Theory of Atoms in Molecules Calculations for Covalent Bonds. J. Phys. Chem. A 2010, 114, 12498-12505.

(51) Fradera, X.; Austen, M. A.; Bader, R. F. W. The Lewis Model and Beyond. J. Phys. Chem. A 1999, 103, 304-314.

(52) Reed, A. E.; Weinstock, R. B.; Weinhold, F. Natural Population Analysis. J. Chem. Phys. 1985, 83, 735-746.

(53) E. D. Glendening, J., K. Badenhoop, A. E. Reed, J. E. Carpenter, J. A. Bohmann, C. M. Morales, C. R. Landis, and F. Weinhold. NBO 6.0; Theoretical Chemistry Institute, University of Wisconsin: Madison, 2013.

(54) Nikolaienko, T. Y.; Bulavin, L. A.; Hovorun, D. M. Janpa: An Open Source Cross-Platform Implementation of the Natural Population Analysis on the Java Platform. Comp. Theor. Chem. 2014, 1050, 15-22.

(55) Fulton, R. L.; Mixon, S. T. Comparison of Covalent Bond Indexes and Sharing Indexes. J. Phys. Chem. 1993, 97, 7530-7534.

(56) Wiberg, K. B. Application of the Pople-Santry-Segal CNDO Method to the Cyclopropylcarbinyl and Cyclobutyl Cation and to Bicyclobutane. Tetrahedron 1968, 24, 1083-1096.

(57) Bader, R. F. W.; Johnson, S.; Tang, T. H.; Popelier, P. L. A. The Electron Pair. J. Phys. Chem. 1996, 100, 15398-15415.

(58) Poater, J.; Fradera, X.; Duran, M.; Solà, M. The Delocalization Index as an Electronic Aromaticity Criterion: Application to a Series of Planar Polycyclic Aromatic Hydrocarbons. Chem. Eur. J. 2003, 9, 400-406. 
(59) Krygowski, T. M.; Bankiewicz, B.; Czarnocki, Z.; Palusiak, M. QuasiAromaticity—What Does It Mean? Tetrahedron 2015, 71, 4895-4908.

(60) Krygowski, T. M.; Ejsmont, K.; Stepień, B. T.; Cyrański, M. K.; Poater, J.; Solà, M. Relation between the Substituent Effect and Aromaticity. J. Org. Chem. 2004, 69, 6634-6640.

(61) Krygowski, T. M.; Szatylowicz, H.; Stasyuk, O. A.; Dominikowska, J.; Palusiak, M. Aromaticity from the Viewpoint of Molecular Geometry: Application to Planar Systems. Chem. Rev. 2014, 114, 6383-6422. 